\title{
Combining Shape from Shading and Stereo: A Variational Approach for the Joint Estimation of Depth, Illumination and Albedo
}

Daniel Maurer

maurer@vis.uni-stuttgart.de

Yong Chul Ju

ju@vis.uni-stuttgart.de

Michael Breuß

breuss@tu-cottbus.de

Andrés Bruhn

bruhn@vis.uni-stuttgart.de
Institute for Visualization and Interactive Systems University of Stuttgart, Germany

Institute for Visualization and Interactive Systems University of Stuttgart, Germany

Institute for Applied Mathematics and Scientific Computing, BTU Cottbus-Senftenberg, Germany

Institute for Visualization and Interactive Systems University of Stuttgart, Germany
Shape from shading (SfS) and stereo are two fundamentally different strategies for imagebased 3-D reconstruction. While approaches for SfS infer the depth solely from pixel intensities, methods for stereo are based on finding correspondences across images.

In this paper we propose a joint variational method that combines the advantages of both strategies. By integrating recent stereo and SfS models into a single minimisation framework, we obtain an approach that exploits shading information to improve upon the reconstruction quality of robust stereo methods. To this end, we fuse a Lambertian SfS approach with a robust stereo model and supplement the resulting energy functional with a detailpreserving anisotropic second-order smoothness term. Moreover, we extend the novel model in such a way that it jointly estimates depth, albedo and illumination. This in turn makes the approach applicable to objects with non-uniform albedo as well as to scenes with unknown illumination.

Experiments for synthetic and real-world images show the advantages of our combined approach: While the stereo part overcomes the albedo-depth ambiguity inherent to all SfS methods, the SfS part improves the degree of details of the reconstruction compared to pure stereo methods. An example of the reconstruction quality of our combined approach using only two views is given in Figure 1. As one can see, the reconstructed depth is quite detailed. Moreover, the computed illumination direction as well as the estimated albedo look reasonable.

[1] C. Strecha, W. von Hansen, L. Van Gool, P. Fua, and U. Thoennessen. On benchmarking camera calibration and multi-view stereo for high resolution imagery. In Proc. IEEE Conference on Computer Vision and Pattern Recognition, 2008.

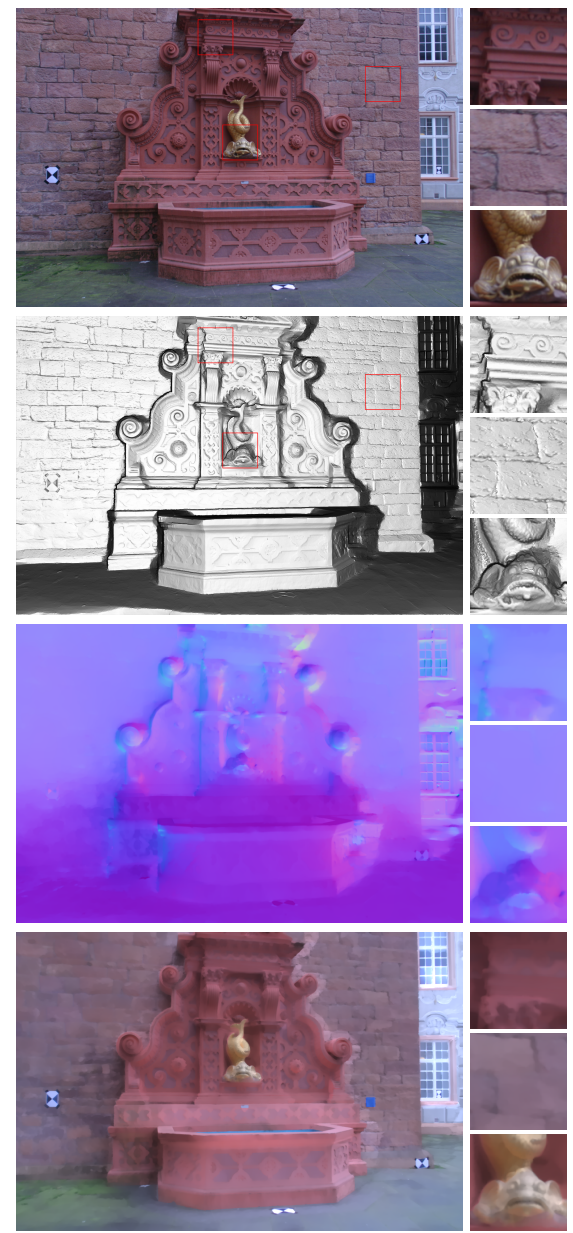

Figure 1: Two-view results for the Fountain-P11 [1]. Top to bottom: Reference image, computed depth (shaded reconstruction), computed illumination direction, computed albedo. 